\title{
Small solar system bodies as granular systems
}

\author{
Daniel Hestroffer ${ }^{1, *}$, Adriano Campo Bagatín ${ }^{2,15}$, Wolfgang Losert ${ }^{3}$, Eric Opsomer ${ }^{4}$, Paul Sánchez ${ }^{5}$, Daniel J. Scheeres ${ }^{6}$, \\ Lydie Staron ${ }^{7}$, Nicolas Taberlet ${ }^{8}$, Hajime Yano ${ }^{9}$, Siegfried Eggl ${ }^{1}$, Charles-Edouard Lecomte ${ }^{7}$, Naomi Murdoch ${ }^{10}$, Fahrang \\ Radjai $^{11}$, Derek C. Richardson ${ }^{12}$, Marcos Salazar ${ }^{13}$, Stephen R. Schwartz ${ }^{14}$, and Paolo Tanga ${ }^{14}$ \\ ${ }^{1}$ IMCCE, Paris Observatory, PSL Research University, CNRS, Sorbonne Univ., UPMC Paris 06, Univ. Lille, F-75014 Paris, France \\ ${ }^{2}$ Departamento de Física, Ingeniería de Sistemas y Teoría de la Señal, Universidad de Alicante, E-03080 Alicante, Spain \\ ${ }^{3}$ Institute for Physical Science and Technology, and Department of Physics, University of Maryland, College Park, MD 20742, USA \\ ${ }^{4}$ GRASP, Research Unit CESAM, University of Liege, Belgium \\ ${ }^{5}$ CCAR, University of Colorado Boulder, Boulder, CO 80309, USA \\ ${ }^{6}$ Aerospace Engineering Sciences, University of Colorado Boulder, Boulder, CO 80309, USA \\ ${ }^{7}$ Inst. Jean Le Rond d'Alembert, Sorbonne Univ, UPMC Univ Paris 06, CNRS, F-75005 Paris, France \\ ${ }^{8}$ Université de Lyon, Laboratoire de Physique, Ecole Normale Supérieure de Lyon, CNRS, F-69364 Lyon, France \\ 9 JAXA/Institute of Space and Astronautical Science, 3-1-1 Yoshinodai, Chuo-ku, Sagamihara, Kanagawa 252-5210, Japan \\ ${ }^{10}$ DEOS/SSPA, ISAE-SUPAERO, Université de Toulouse, F-31055 Toulouse, France \\ ${ }^{11}$ LMGC, Université de Montpellier, CNRS, Montpellier, France \\ ${ }^{12}$ Department of Astronomy, University of Maryland, College Park, MD 20742-2421, USA \\ ${ }^{13}$ LICB, Univ. de Bourgogne, CNRS, F21078 Dijon, France \\ ${ }^{14}$ Université Côte d'Azur, OCA, CNRS, IRD, Laboratoire Lagrange, F-06304 Nice, France \\ ${ }^{15}$ Instituto Universitario de Física Aplicada a las Ciencias y la Tecnología, Universidad de Alicante, E-03080 Alicante, Spain
}

\begin{abstract}
Asteroids and other Small Solar System Bodies (SSSBs) are currently of great scientific and even industrial interest. Asteroids exist as the permanent record of the formation of the Solar System and therefore hold many clues to its understanding as a whole, as well as insights into the formation of planetary bodies. Additionally, SSSBs are being investigated in the context of impact risks for the Earth, space situational awareness and their possible industrial exploitation (asteroid mining). In all these aspects, the knowledge of the geophysical characteristics of SSSB surface and internal structure are of great importance. Given their size, constitution, and the evidence that many SSSBs are not simple monoliths, these bodies should be studied and modelled as self-gravitating granular systems in general, or as granular systems in micro-gravity environments in particular contexts. As such, the study of the geophysical characteristics of SSSBs is a multi-disciplinary effort that lies at the crossroads between Granular Mechanics, Celestial Mechanics, Soil Mechanics, Aerospace Engineering and Computer Sciences.
\end{abstract}

\section{Introduction}

Long seen as a point-like source in the sky, asteroids first discovered in the $19^{\text {th }}$ Century - are Solar System bodies named after their star-like appearance. More recent ground-based and space probe observations have revealed that asteroids have to be considered as small worlds on their own, some even forming systems with satellites. Those small Solar System bodies (SSSBs; comets, asteroids, meteoroids) orbiting around the Sun are found at different heliocentric distances ranging from close-by Near Earth Objects, between Mars and Jupiter, and beyond Neptune. The distinction between asteroids and comets, when analysing their physical properties is not very sharp. The transition, referred to as the "asteroid-comet continuum" shows that both have similar constituents, with likely larger proportion of volatiles for icy comets (those that are sublimated during passage at perihelion) than for rocky asteroids with higher concentration of carbon, silicates, and metals (that sometimes show some cometary-like activity). Due to their small sizes, the SSSBs are the pristine remnants of the formation of the Solar System, and hence important tracers of the history of planet formation, the evolution of the planetary system, and possibly for the origin of life on Earth. It is then of prime importance to better understand their constitution, as individual objects, and on a global basis as a system of objects. To this effect different techniques are used to characterise the physical properties of their surface and interior, and how they evolve on astronomical time scales due to dynamical perturbations, collisions, physical process, etc. One of the main features that has been revealed in the last decades is that small bodies can have low bulk densities compatible with "rubble pile" structures or gravitational aggregates [1], with low cohesion, and sustained plastic deformation. These bodies also show a large variety of irregular shape morphology, macro-porosity, surface properties (from smooth regolith covered terrain, to very rough collection of boulders), and topography (cratering,

\footnotetext{
Corresponding author: hestro@imcce.fr
} 
depressions, ridges) - see Fig. 1. All this has generated many studies and modelling to better understand the macro- and micro-scale structure and behaviour of these bodies, their formation and evolution, etc. In particular, as shown here, some key problems of mechanics and nonlinear dynamics of self-gravitating celestial bodies can best be approached by granular media modelling. A comprehensive review of asteroids and asteroid science was recently completed for the book Asteroids IV [2].

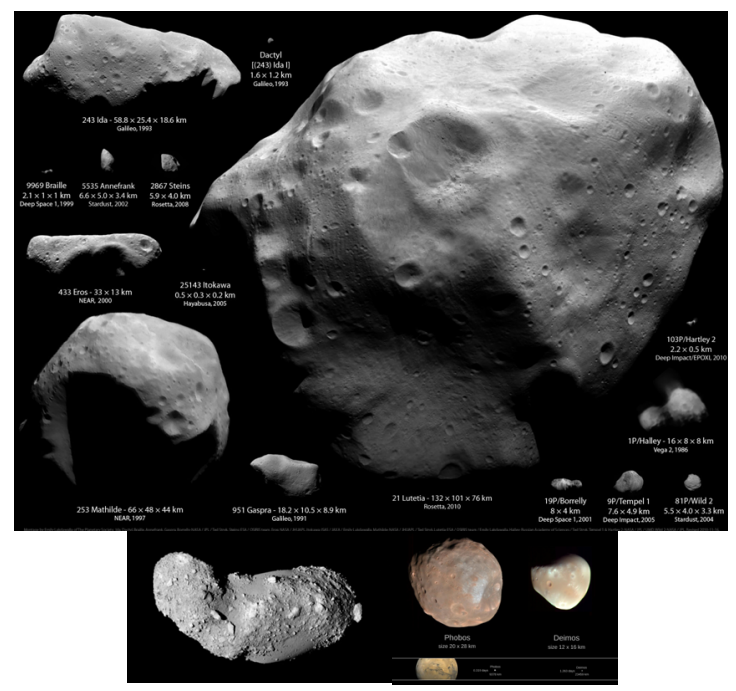

Fig. 1. Collage of images from various space missions to asteroids, comets, and satellites (see Table 1). The largest (21) Lutetia around $100 \mathrm{~km}$ diameter dominates its neighbours (top); the smallest (25143) Itokawa around $0.5 \mathrm{~km}$ size is enlarged (bottom left); the two Martian moons are added (bottom right). Credits: E. Lakdawalla (the Planetary Society), JAXA, ESA, NASA.

\section{Mission and observations}

The fundamental idea for why granular mechanics is relevant for the study of SSSBs emerged during space missions to visit these bodies. Theories developed in the seventies and eighties for the properties of SSSBs generally imagined asteroidal bodies to be comprised of large monolithic blocks with little or no surface covering of finer material (regolith). This changed drastically given the initial observations of asteroids Gaspra and Ida by the Galileo spacecraft in the early 1990's, the first direct images of such bodies. Snapshot images taken of these asteroids showed them to be covered with surface regolith, to be heavily cratered, and in the case of Ida to have a binary asteroid companion which itself had a regolith covering. Since these initial observations the space-based and ground-based observations of asteroids have grown, along with the realization that these bodies are fundamentally granular in nature and hence that their physical evolution must be described using principles of granular mechanics applied to these extreme environments.

There are a few key missions and ground-based observations that have shaped our view of these bodies as having a granular nature. Table 1 below presents a comprehensive list of asteroids visited by spacecraft.
Table 1. List of achieved space missions to small bodies.

\begin{tabular}{|c|c|c|}
\hline Target & Mission & Date \\
\hline \multicolumn{3}{|l|}{ Asteroids } \\
\hline (4179) Toutatis & Chang'E 2 & Dec. 2012 \\
\hline (21) Lutetia & Rosetta & July 2010 \\
\hline (2867) Steins & Rosetta & Sep. 2008 \\
\hline (25143) Itokawa & Hayabusa & June 2005 \\
\hline (5535) Annefrank & Stardust & Nov. 2002 \\
\hline (433) Eros & NEAR Shoemaker & Feb. 2000 \\
\hline (9969) Braille & Deep Space 1 & Jan. 1999 \\
\hline (253) Mathilde & NEAR Shoemaker & June 1997 \\
\hline (243) Ida & Galileo & Aug. 1993 \\
\hline (951) Gaspra & Galileo & Oct. 1991 \\
\hline \multicolumn{3}{|l|}{ Comets } \\
\hline $\begin{array}{l}\text { 67P/Churyumov- } \\
\text { Gerasimenko. }\end{array}$ & Rosetta & Aug. 2014 \\
\hline 103P/Hartley2 & EPOXI & Nov. 2010 \\
\hline Tempel 1 & Deep Impact, Stardust & Jul. 2005, 2011 \\
\hline Wild 2 & Stardust & Jan. 2004 \\
\hline 19P/Borrelly & DS 1 & Sep. 2001 \\
\hline Halley & $\begin{array}{l}\text { Giotto, Vega } 1 \& 2 \text {, Sakigake, } \\
\text { Suisei, ICE }\end{array}$ & March 1986, \\
\hline Giacobini-Zinner & ICE & Sep. 1985 \\
\hline \multicolumn{3}{|l|}{ Moons } \\
\hline M1 Phobos & $\begin{array}{l}\text { MEX, ODY, MGS, Phobos2, } \\
\text { Viking1 }\end{array}$ & $\begin{array}{l}2004,2001, \\
1997,1989, \\
1976\end{array}$ \\
\hline M2 Deimos & MEX, ODY, MGS, Viking2 & $\begin{array}{l}2004,2001 \\
1997,1976\end{array}$ \\
\hline
\end{tabular}

NEAR Mission: NASA's NEAR Shoemaker mission visited two asteroids, with a fast flyby of the large asteroid (253) Mathilde en route to a year-long orbital mission about the asteroid (433) Eros [3,4]. The flyby of Mathilde was notable as it was the first direct mass measurement of an asteroid and, when included with an estimate of the total volume of that asteroid, yielded a bulk density of $1300 \mathrm{~kg} / \mathrm{m}^{3}$ that was surprisingly low for a C-type asteroid, and consistent with a highly porous structure. This was the first direct evidence of a high porosity within asteroids, consistent with a rubble pile structure. Asteroid Eros was observed at high resolution for an extended period of time, with the mission ending with a controlled landing to the asteroid surface, providing high-resolution imaging of the body. These observations showed the surface to be covered with a thick layering of regolith, and with the crater density of the surface virtually disappearing at small size scales, seemingly obliterated by resurfacing processes that moved the regolith across the surface. This has since been attributed to seismic shaking of the body due to cratering impacts that can resurface the body. Another 
feature of the surface was the presence of many areas of "ponded" material, consisting of finer regolith being preferentially trapped at the bottom of craters, in some cases partially embedding boulders. The transport of this material is not fully understood, but could be due to electrostatic levitation of dust fines which are then transported laterally over the surface to local geopotential lows. The presence of the fine regolith is attributed to the ability of the large $(\approx 11 \mathrm{~km})$ NEO Eros to retain more of the fine regolith after an impact.

Hayabusa Misson: The Japanese space agency's (JAXA's) Hayabusa mission visited asteroid Itokawa in 2005. The mission mostly focused on a 3-month period of characterization and sampling attempts. Due to its much smaller size, $(\approx 0.5 \mathrm{~km})$, not as much fine regolith is retained on this body, providing a clear view of its fundamentally rocky and block-covered surface. Despite this, there are specific regions where finer regolith has been seen to flow across the surface into the geopotential lows of the body. The observed boulders on the surface of Itokawa have been documented and determined to follow a roughly $1 / d^{3}$ size distribution, which corresponds to an equal mass distribution for every size, with the largest boulder being on the order of a few decameters. Also observed were larger boulders of indeterminate length thrusting out of the interior of the asteroid. The asteroid probe also collected a small sample, which was seen to consist of a particle size distribution $1 / d^{x}$ with $x$ between 2 and 2.7. The body was also determined to have a high porosity of approximately $40 \%[5,6]$.

Ground-Based Observations: Evidence for the granular nature of small asteroids has also come from groundbased telescopic observations of these bodies. A key, and early, indication came from the statistics of asteroid spin rates vs. size, which showed that at small sizes the spin rates of asteroids is higher, indicating that these may be components of larger asteroids that could not spin as fast without falling apart. Also, binary asteroids which provide insight into the masses of these systems generally confirm the high porosity seen from space missions. Another source of insight comes from the shape models developed using range-Doppler imaging of asteroids. The accuracy of these models is sufficient to develop high resolution, non-convex shape models that provide insight into the surface slope distributions of these bodies. It has generically been found that these bodies all have the vast majority of their surface slopes below $35^{\circ}$, the usual angle of repose for granular materials, indicating surfaces that are likely covered with some sort of granular material. In at least one case however - for the asteroid 1950 DA - this is not true, the mass determination of this body indicates that it is highly porous and fast spinning, implying that this body has some level of cohesive strength. Other lines of evidence discovered recently include asteroid pairs and their link to rotational fission models and active asteroids which are seen to separate into multiple components.
Current Questions and Hypotheses: These recent observations have strengthened the concept of granular mechanics being important for the physical evolution and properties of these asteroids. Most asteroids smaller than $100 \mathrm{~km}$ are likely gravitational aggregates [7], which implies that they can be considered as large-scale granular structures, where grains are components of tens to hundreds of meters in size. The same applies to surfaces with grains of smaller sizes. Based on these ideas there have been a number of hypotheses and models for the behaviour of asteroids motivated by granular mechanics. First and foremost, the "Brazil nut effect" is invoked to hypothesise the percolation of larger boulders and grains to the asteroid surface. Other theories include a cohesion theory that posits the presence of cohesive strength in rubble pile asteroids arising from weak van der Waals forces between the finest components of asteroid regolith. Predictions from that theory have been seen to be consistent with constraints on cohesive strength within asteroids based on ground observations. These theories have, in turn, been tied to a range of possible models of internal porosity distribution within asteroids that lead to morphologies of different strength: depending on whether the interior of a gravitational aggregate has additional strength or has less strength (due to lower, or higher porosity), different failure patterns are expected to occur when the body is spun to high spin rates.

\section{Numerical simulations}

There are three main numerical methods that have been applied to the study of asteroid surfaces and interiors [8, and ref. therein]: Soft-sphere Discrete Element Method (SSDEM) [e.g. 9,10], Hard-sphere Discrete Element Method (HSDEM) [e.g. 11], and Finite Element Method (FEM) [e.g. 12].

FEM simulation codes, together with Soil Mechanics theory, model asteroids as a continuum in which the sizes of the constituent particles are much smaller than the characteristic length of the system. Here the geophysical characteristics are an input to the codes and system behaviour is most affected by the choice of yield criterion (Mohr-Coulomb, Drucker-Prager, etc.). Discrete methods model asteroids as a collection of selfgravitating particles (generally spherical) that conserve some of the geophysical characteristics of the bodies to be studied (angle of friction, cohesive strength, bulk density, etc.). These characteristics are the result of the interaction of individual grains and are, therefore, an output of the simulations that correspond to the material parameters of the grain-grain interaction models. Softsphere DEM codes integrate the equations of motion of every grain and allow them to overlap; it is this overlap that provides a repulsive force during collisions and sustained contact. Hard-sphere HSDEM avoids this overlap by transferring momentum in every collision and assuming it to be instantaneous. This makes it much faster than SSDEM, applicable to very kinetically active systems but not applicable to systems where sustained contacts are necessary. On the other hand, FEM codes are less computationally intensive, and can easily deal 
with realistic asteroid shapes and mass, but they can only show a static picture of the body, where the behaviour of the aggregate is assumed to follow a yield criterion. DEM simulations are far more computationally intensive, but have the advantage that they show a dynamical evolution and the material parameters are generally known and implemented.

In addition to topics common to granular mechanics research such as particle size segregation, stress states, and penetrometry, the granular physics of asteroids raises several unique challenges including: rotation induced deformation and disruption, collisional evolution, interior heterogeneities, gravitational tide induced deformation, interior heterogeneities, cratering, and sampling mechanisms, among the most important $[12,13]$.

\section{Laboratory experiments}

To address the specific context of planetary science, the classical laboratory experiments of granular mechanics need to be adapted to micro-gravity or low-gravity and vacuum space. Thus, tests are generally performed in drop towers, in parabolic or ballistic flights, or on the international space station (see chart below). Unlike drop-towers or sounding rockets, parabolic flights (PF) enable the presence of scientists operating the experiment. The latter is of particular interest for studies requiring human interactions and manipulations. $\mathrm{PF}$ are adapted to short-duration ( $20 \mathrm{~s})$ scientific and technological investigations in low-gravity. An example for PF experimentation in the field of granular matter is given by ESA's VIP-Gran instrument, which is designed for the study of the dynamics and the statistical mechanics of an ensemble of particles that interact through dissipative collisions. During ESA's PFC65 flight campaign, the segregation of driven binary granular media through vibrations was investigated within the frame of VIP-Gran. For specific filling conditions, different dynamical regimes as well as segregation of both species were reported $[14,15]$. In another set of PF experiments, it was demonstrated that convective-like flows in a granular material are close to zero in microgravity [16]. As it has been observed, one of the main issues with the previous experimental methodologies is their duration. In order to avoid this, the Strata-1 experiment is being carried out for 1 year in the International Space Station [17]. This experiment consists of 4 tubes that contain a different granular material of a known size distribution in each one. The objective is to observe how the grains mix and segregate in an extremely low-gravity environment under gentle vibration during an extended period of time.

\section{Future prospect and opportunities}

Currently on the horizon are two space missions to small, primitive asteroids believed to have a granular "rubble pile" structure. These are the Japanese Hayabusa-2 mission to asteroid Ryugu and NASA's OSIRIS-REx mission to asteroid Bennu. Both of these missions have been launched and are en route to their targets. Hayabusa2 will arrive in the summer of 2018 and OSIRIS-REx in the late fall of 2018. Both will spend at least one year at their respective target bodies before coming back to Earth with surface samples. Data from both missions, numerical simulations, and laboratory experiments can be used to further probe the granular nature of their target bodies, and to further deepen the ties between granular mechanics and asteroid geophysics.

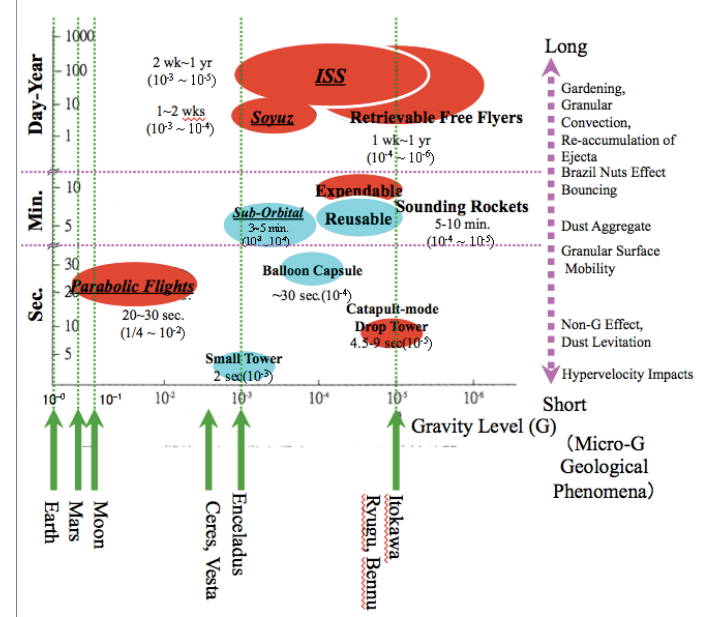

Fig. 2. Summary of the micro-gravity experiment facilities.

The authors acknowledge support by the International Space Science Institute (ISSI, Bern, Switzerland) and Paris observatory (France) to the international team «Asteroids \& Self-Gravitating Bodies as Granular Systems ${ }^{a} »$. EO acknowledges European Space Agency program SpaceGrains and Prodex (Belspo, Brussels, Belgium) for financial support.

\section{References}

1. D.C. Richardson et al. In Asteroids III, p. 501 (2002)

2. Asteroids IV. Eds. P. Michel, F.E. DeMeo, W.F. Bottke. 785 pp. The Univ. of Arizona Press (2015)

3. A.F. Cheng. AdSR 33, 1558 (2004)

4. J. Veverka et al. Science 278, 2109 (1999)

5. A. Fujiwara et al. Science 312, 1330 (2006)

6. H. Yano et al. Science 312, 1350 (2006)

7. A. Campo Bagatin et al. Icarus 149, 198 (2001)

8. N. Murdoch et al. In Asteroids IV, p. 767 (2015)

9. C. Maurel et al. MNRAS 464, 2866 (2017)

10. P. Sánchez \& D.J. Scheeres. ApJ 727, 120 (2011)

11. D.C. Richardson et al. Icarus 143, 45 (2000)

12. M. Hirabayashi \& D.J Scheeres. ApJ 798, L8 (2015)

13. D.J. Scheeres et al. In Asteroids IV, p. 745 (2015)

14. E. Opsomer, et al. Eur. Phys. J. E 37, 115 (2014)

15. More information on http://spacegrains.org

16. N. Murdoch et al. 2013. PRL 110, 018307 (2013)

17. M. Fries et al. LPIContrib. 1921, 6547 (2016)

\footnotetext{
${ }^{a}$ ISSI team http://www.issibern.ch/teams/granularsystems/
} 